\title{
Simulation of SU(2) gauge theory with improved domain-wall fermions
}

\author{
Hideo Matsufuru*i \\ High Energy Accelerator Research Organization (KEK), Tsukuba 305-0801, Japan \\ E-mail: hideo.matsufuruekek.jp \\ Norikazu Yamada \\ High Energy Accelerator Research Organization (KEK), Tsukuba 305-0801, Japan \\ E-mail: norikazu.vamadadkek.ip
}

\begin{abstract}
We investigate the $\mathrm{SU}(2)$ gauge theory with the domain-wall fermions. Our previous simulations with many flavors of the standard domain-wall fermions suffered from rather large residual mass that obstruct the study in the small mass region. To improve the domain-wall fermion action, we employ the link smearing technique that consists of the HYP smearing and the stout projection. On the previously generated configurations with the two flavors of the standard domain-wall fermions, we confirm that the improved domain-wall fermion operator indeed reduce the residual mass to a factor of five. Dynamical simulations with two flavors of improved domain-wall fermions are in progress.
\end{abstract}

34th annual International Symposium on Lattice Field Theory

24-30 July 2016

University of Southampton, UK

* Speaker.

${ }^{\dagger}$ A footnote may follow. 


\section{Introduction}

The SU(2) gauge theory has recently been drawn much attention in search for a theory beyond the standard model as a candidate of technicolor theory [四]. It is also interesting from a view point of the spontaneous chiral symmetry breaking, since the symmetry breaking pattern of the $\mathrm{SU}(2)$ gauge theory with fundamental fermions $S U\left(2 N_{f}\right) \rightarrow S p\left(2 N_{f}\right)$ is different from that of QCD, $S U\left(N_{f}\right) \times S U\left(N_{f}\right) \rightarrow S U\left(N_{f}\right)$, where $N_{f}$ is the number of flavors. To explore the chiral symmetry on the lattice, it is important to employ the fermion formulation that retains the chiral symmetry as exact as possible. Although the overlap fermions hold the exact chiral symmetry, because of its large numerical cost, in this study we adopt the domain-wall fermions as dynamical degrees of freedom and apply to numerical simulations with many flavors.

Our previous work with the standard domain-wall fermions [ [ $\left[\right.$, []] showed that for $N_{f}=2,4$, the systems are characterized by spontaneously broken chiral symmetry as same as QCD, while for $N_{f}=8$ such a feature seems to disappear in the massless limit. For more detailed analysis, in particular to clarify the marginal case of $N_{f}=6$, it is necessary to explore the vicinity of the massless limit. However, the rather large residual mass of the standard domain-wall fermion makes such analysis difficult. To access the small fermion mass regime, it is necessary to employ an improved domain-wall action such as the Möbius formulation [四], the optimal domain-wall operator [四], or the link smearing [ [G, 四]. To reduce the residual mass, it is efficient to reduce the low-lying eigenvalue density of the hermitian Wilson kernel in the domain-wall operator. Since this may be achieved by employing the link smearing technique, in this work we apply the link smearing to the standard form of the domain-wall fermion action.

In the next section we introduce the improved domain-wall fermion with the link smearing, and in Section 3 we examine the residual mass on the gauge configurations previously generated with the unimproved domain-wall fermions. Section $⿴$ describes the dynamical simulations with two flavors. The last section gives our conclusion and outlook.

\section{Improved domain-wall fermion}

The domain-wall fermion operator is represented as

$$
\begin{aligned}
D_{D W}\left(x, s ; y, s^{\prime}\right)=D_{W}(x, y) \delta_{s, s^{\prime}} & -\frac{1}{2}\left[\left(1-\gamma_{5}\right) \delta_{x, y} \delta_{s+1, s^{\prime}}+\left(1+\gamma_{5}\right) \delta_{x, y} \delta_{s-1, s^{\prime}}-2 \delta_{x, y} \delta_{s, s^{\prime}}\right] \\
+ & m\left[P_{R} \delta_{x, y} \delta_{s, 1} \delta_{s^{\prime}, L_{s}}+P_{L} \delta_{x, y} \delta_{s, L_{s}} \delta_{s^{\prime}, 1}\right]
\end{aligned}
$$

where $m$ is fermion mass, $P_{R, L}=\left(1 \pm \gamma_{5}\right) / 2, L_{s}$ the size of 5th dimension, and $D_{W}$ the standard Wilson kernel with the negative mass $-M_{0}$ (the domain-wall height),

$$
D_{W}[U](x, y)=4-M_{0}+\kappa \sum_{\mu}\left[\left(1-\gamma_{\mu}\right) U_{\mu}(x) \delta_{x+\hat{\mu}, y}\left(1-\gamma_{\mu}\right) U_{\mu}(x-\hat{\mu}) \delta_{x-\hat{\mu}, y}\right] .
$$

As an improvement scheme, we adopt the HYP link smearing [ [G, $\square]$ combined with the stout exponentiation of the smeared link [ $[\mathrm{d}]$. The smeared domain-wall fermion action is obtained by replacing $U_{\mu} \rightarrow U_{\mu}^{\mathrm{smr}}$ in Eq. ([2.2). The link smearing consists of a scheme to incorporate the extended paths of links, $C_{\mu}(x)$, and a method to recast it into the gauge group, $P\left[\alpha ; C_{\mu}(x), U_{\mu}(x)\right]$. The 
HYP smearing scheme recursively sums over staples so that the links in the adjacent hypercubes to the original link are incorporated [目],

$$
\begin{aligned}
& V_{\mu ; v \rho}^{(1)}(x)=P\left[\alpha_{3} ; C_{\mu ; v \rho}^{(1)}(x), U_{\mu}(x)\right], \quad C_{\mu ; v \rho}^{(1)}(x)=\frac{1}{2} \sum_{ \pm \sigma \neq \mu, v, \rho} U_{\sigma}(x) U_{\mu}(x+\hat{\sigma}) U_{\sigma}^{\dagger}(x+\hat{\mu}) \\
& V_{\mu ; v}^{(2)}(x)=P\left[\alpha_{2} ; C_{\mu ; \nu}^{(2)}(x), U_{\mu}(x)\right], \quad C_{\mu ; \nu}^{(2)}(x)=\frac{1}{4} \sum_{ \pm \rho \neq \mu, v} V_{\rho ; \mu \nu}^{(1)}(x) V_{\mu ; v \rho}^{(1)}(x+\hat{\rho}) V_{\rho ; \mu \nu}^{(1) \dagger}(x+\hat{\mu}) \\
& V_{\mu}(x)=P\left[\alpha_{1} ; C_{\mu}^{(3)}(x), U_{\mu}(x)\right], \quad C_{\mu}^{(3)}(x)=\frac{1}{6} \sum_{ \pm v \neq \mu} V_{v ; \mu}^{(2)}(x) V_{\mu ; \nu}^{(2)}(x+\hat{v}) V_{v ; \mu}^{(2) \dagger}(x+\hat{\mu}) .
\end{aligned}
$$

As the projection method, the stout projection [ [8]

$$
P\left[\alpha ; C_{\mu}(x), U_{\mu}(x)\right]=\exp \left(\alpha\left[C_{\mu}(x) U_{\mu}^{\dagger}(x)\right]_{A T}\right) U_{\mu}(x)
$$

is employed. For $\mathrm{SU}(2)$ matrix $Q$, the Cayley-Hamilton theorem reads $Q^{2}-c_{0} I=0$ where $c_{0}=$ $\operatorname{det} Q=\frac{1}{2} \operatorname{tr} Q^{2} \geq 0$ and thus

$$
e^{i Q}=f_{0}\left(c_{0}\right)+f_{1}\left(c_{0}\right) Q
$$

holds, where $f_{j}$ 's are the functions of $c_{0}$. The analytic form of the HMC force is derived with a little modification to the SU(3) case. The link smearing is recursively applicable. We recursively apply the smearing procedure Eq. ([2.3) twice, and in the following, this improved domain-wall fermion is denoted by the stout-HYP(2) domain-wall or the HYP-DW fermion in short.

\section{Effect of link smearing}

We first examine the effect of improvement for the valence domain-wall fermions on the configuration ensembles that were previously generated with the Iwasaki gauge action and two flavors of the standard domain-wall fermions [0] $]$. These configurations were generated on a $16^{3} \times 32$ with $M_{0}=1.6, L_{s}=16$, and at several values of $\beta$ and fermion mass $m$. The valence fermion is the stout-HYP(2) domain-wall action with $L_{s}=16$ and $m_{\text {val }}=0.010$. The smearing parameters are set to $\alpha_{1}=0.95, \alpha_{2}=0.76$, and $\alpha_{3}=0.38$ [可].

Figure $\square$ shows the $M_{0}$ dependence of the residual mass for the HYP-DW action on the ensembles generated at $(\beta, m)=(0.85,0.050)$ and $(0.90,0.050)$. In each ensemble, 80 configurations are used for the measurements. The residual mass is indeed reduced by about factor 5 compared to the unimproved domain-wall fermions: $m_{\text {res }} \simeq 0.030$ (at $\beta=0.85$ ), and $m_{\text {res }} \simeq 0.013(\beta=0.90$ ). At both the values of $\beta$, the residual mass takes the minimum value around $M_{0}=1.6$ with mild $M_{0}$ dependence around this value.

The residual mass is related to the low-lying eigenvalue density of the hermitian Wilson kernel $H_{W}=\gamma_{5} D_{W}$ [प]]. Figure $\square$ displays the eigenvalue distributions of the Wilson kernel with $M_{0}=1.6$ with and without the link smearing, respectively. The link smearing indeed reduces the low-lying eigenvalue density of $H_{W}$ by about factor 5 .

\section{Dynamical simulations}

Motivated by the results in the last section, we perform dynamical simulations with two flavors of the stout-HYP(2) smeared domain-wall fermions on a $16^{3} \times 32$ lattice. We adopt the Iwasaki 

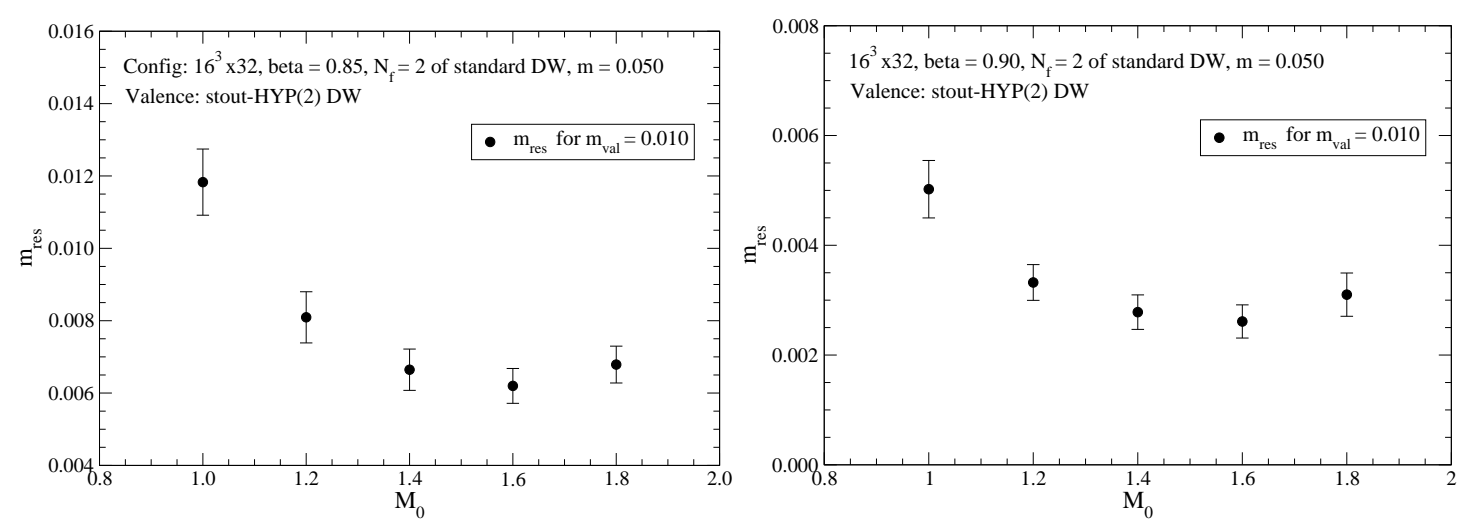

Figure 1: The $M_{0}$ dependence of the residual mass for the valence stout-HYP(2) DW fermion operators on $N_{f}=2$ configurations generated with the standard DW fermions.
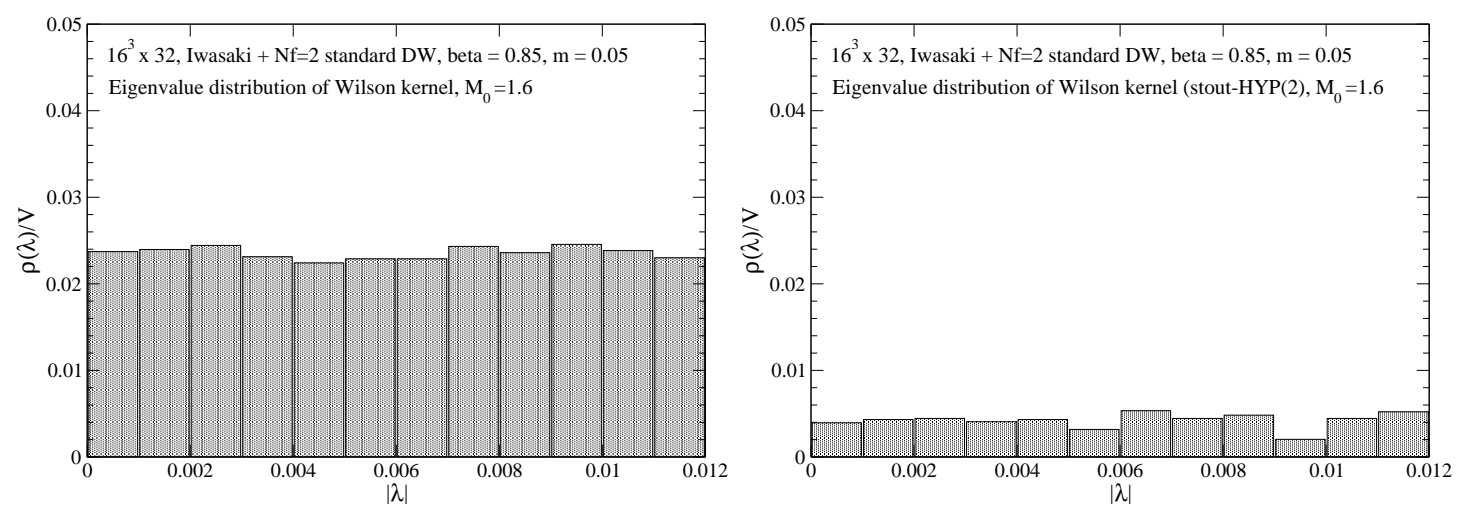

Figure 2: Eigenvalue distribution on $16^{3} \times 32$ lattice with $\beta=0.85, N_{f}=2, m_{\text {sea }}=0.05$. Left and right panels display the eigenvalue distributions of the Wilson kernel with and without the link smearing, respectively.

gauge action and set $L_{s}=16, M_{0}=1.6$, and the smearing parameters $\alpha_{1}=0.95, \alpha_{2}=0.76$, and $\alpha_{3}=0.38$, as same as the previous section. The gauge configurations are generated at $\beta=0.90$ with five values of dynamical fermion masses, $m_{\text {sea }}=0.002,0.005,0.010,0.020$, and 0.050 . The standard hybrid Monte Carlo algorithm is applied with unit trajectory length. Currently $O(1000)$ trajectories are compiled at each parameter set.

We first measure the Wilson loops at every five trajectories and extract the static fermion potential. The left panel of Fig. [3 shows the fermion mass dependence of the lattice scale set by the hadronic radius $r_{0}$ by setting $r_{0}=0.49 \mathrm{fm}$. The result shows mild dependence of $r_{0}$ on the fermion mass $m$, and the lattice spacing $a$ smaller than the corresponding result of the standard domain-wall fermions. The right panel of Fig. [ 3 displays the residual mass $m_{\text {res }}$ of the domain-wall fermions. The residual mass is measured at the valence mass equal to the sea fermion mass. As expected, the values of $m_{\text {res }}$ are significantly reduced by the link smearing.

On each dynamical ensemble, the meson mass is measured for several valence fermion masses. We first measure the meson correlators with local operators at the source and sink. Figure $\$$ shows the valence fermion mass dependence of the squared pseudoscalar meson mass $m_{P S}$ on ensembles of $m_{\text {sea }}=0.010$ and 0.050 . The valence fermion mass includes the residual mass measured at each 

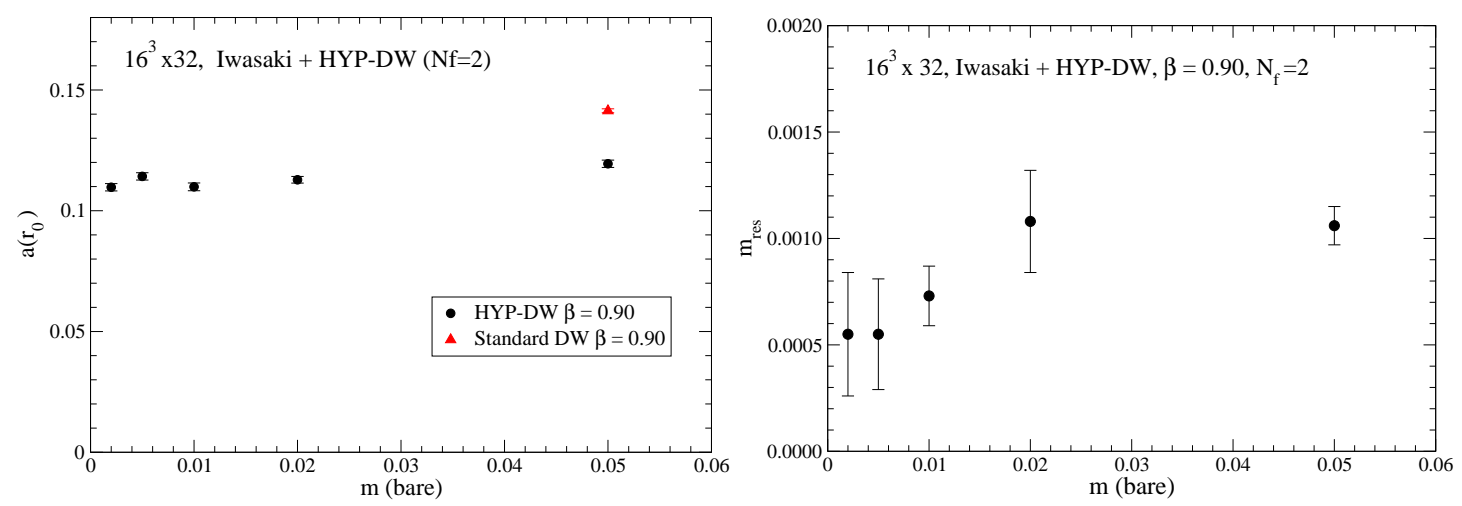

Figure 3: The results of dynamical simulation with two flavors of stout-HYP(2) domain-wall fermions on $16^{3} \times 32$ at $\beta=0.90$. The left panel displays the lattice scale set by $r_{0}$ from the static potential. In addition to the value at each bare dynamical fermion mass, we include the result of the standard domain-wall fermion simulation. The right panel shows the residual masses measured at $m_{\mathrm{val}}=m_{\mathrm{sea}}$.

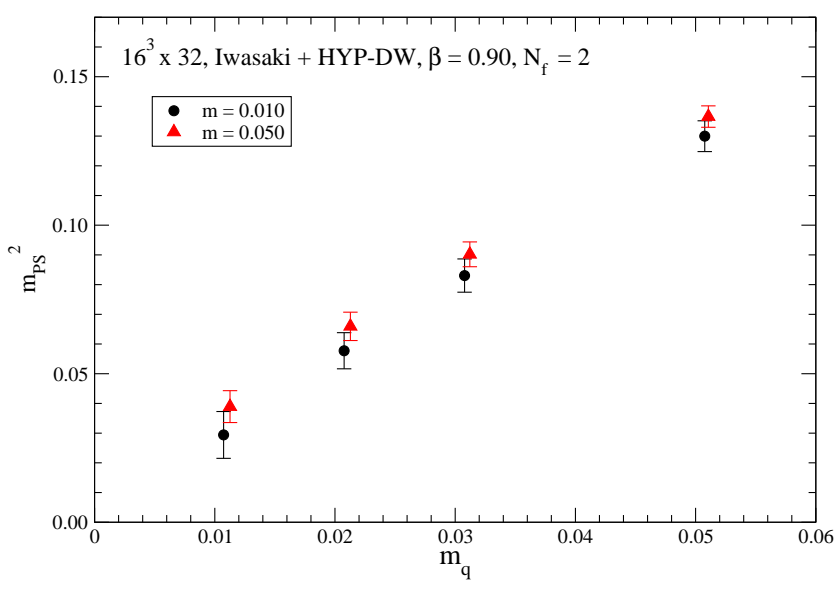

Figure 4: The results of pseudoscalar meson spectrum on dynamical configurations with two flavors of stout-HYP(2) domain-wall fermions on $16^{3} \times 32$ at $\beta=0.90$. On $m_{\text {sea }}=0.010$ and 0.050 ensembles, $m_{P S}^{2}$ is plotted against the valence fermion masses including the residual mass, $m_{q}=m_{\mathrm{val}}+m_{\mathrm{res}}$.

value. Both the results show a linear dependence of the $m_{P S}^{2}$ on the fermion mass. While the result of $m_{\text {sea }}=0.050$ exhibits small intercept at the massless limit, it disappears on the $m_{\text {sea }}=0.010$ ensemble. More detailed analysis is in progress.

\section{Conclusion and outlook}

To explore the small fermion mass region, we investigated the domain-wall fermion improved by the link smearing. Applying the stout-HYP smearing twice to the link variable in the fermion operator, we observed that the residual mass is significantly reduced compared to the unimproved domain-wall operator. Application to the $N_{f}>2$ cases is underway. It is also straightforward to apply to the adjoint domain-wall fermion [B]].

The spectrum result of dynamical simulation with two flavors indicates that by setting the fermion mass sufficiently small the $\varepsilon$-regime simulation, in which the pion Compton wavelength is much larger than the system size, may be possible with e.g. adopting $L=8$ lattice. Comparison 
of the eigenvalue spectrum to the prediction of the random matrix theory is important [W]]. Such a study is also in progress.

\section{Acknowledgment}

Numerical simulations were performed on Hitachi SR16000 and IBM Blue Gene/Q at KEK under a support of its Large-scale Simulation Program (Nos. 14/15-22, 15/16-14), and $\varphi$ computer system at Kobayashi-Maskawa Institute, Nagoya University. The gauge ensembles used in this work were generated in collaboration with K.-i. Nagai. We also thank the Japan Lattice Data Grid which is a grid file system constructed on a virtual private network HEPnet-J/sc on SINET provided by National Institute of Informatics for efficient data transfer. The simulation codes used in this work is based on the Bridge++ code set [प2]. This work is supported in part by the Grand-in-Aid for Scientific Research of Japan (Nos.22224003, 22740183, 25400284, 15H03669).

\section{References}

[1] For reviews, J. Kuti, PoS LATTICE 2013 (2014) 004; E. T. Neil, PoS LATTICE 2011 (2011) 009 [arXiv:1205.4706 [hep-lat]]; J. Giedt, PoS LATTICE 2012 (2012) 006.

[2] H. Matsufuru, K. i. Nagai and N. Yamada, PoS LATTICE 2014, 241 (2014);

[3] H. Matsufuru, K. I. Nagai and N. Yamada, PoS LATTICE 2015 (2016) 054.

[4] R. C. Brower, H. Neff and K. Orginos, Nucl. Phys. Proc. Suppl. 140 (2005) 686 doi:10.1016/j.nuclphysbps.2004.11.180 [hep-lat/0409118].

[5] T. W. Chiu, Phys. Rev. Lett. 90 (2003) 071601 doi:10.1103/PhysRevLett.90.071601 [hep-lat/0209153].

[6] A. Hasenfratz and F. Knechtli, Phys. Rev. D 64 (2001) 034504 doi:10.1103/PhysRevD.64.034504 [hep-lat/0103029].

[7] A. Hasenfratz, R. Hoffmann and S. Schaefer, JHEP 0705 (2007) 029 doi:10.1088/1126-6708/2007/05/029 [hep-lat/0702028].

[8] C. Morningstar and M. J. Peardon, Phys. Rev. D 69 (2004) 054501 doi:10.1103/PhysRevD.69.054501 [hep-lat/0311018].

[9] S. Durr et al., JHEP 1108 (2011) 148 [arXiv:1011.2711 [hep-lat]].

[10] R. Narayanan and H. Neuberger, Nucl. Phys. B 412 (1994) 574 doi:10.1016/0550-3213(94)90393-X [hep-lat/9307006].

[11] P. H. Damgaard and S. M. Nishigaki, Phys. Rev. D 63 (2001) 045012 [hep-th/0006111].

[12] S. Ueda et al., PoS LATTICE 2013, 412 (2014); J. Phys. Conf. Ser. 523, 012046 (2014). 